\title{
CONVERGENCE IN MEASURE AND RELATED RESULTS IN FINITE RINGS OF OPERATORS
}

BY

\author{
A. R. PADMANABHAN
}

Dedicated to Professor Ganapathy Iyer on his sixtieth birthday

Introduction. The foundations of a noncommutative integration theory were laid by Segal in [6], in connection with investigations in quantum mechanics, operator algebras and harmonic analysis on groups. In the aforesaid disciplines, there arise systems which are (noncommutative) analogues of measurable functions on a conventional measure space, the simplest instances of such systems being the so-called factors of type $\mathrm{I}_{n}$, e.g., the ring of all complex $n \times n$ matrices together with the trace. If the trace is normalized so as to assume the value unity, on the identity operator, then this system becomes the noncommutative analogue of complex random variables, on a probability space generated by $n$ atoms.

Let $G$ be a locally compact unimodular group (such as, for some fixed $n$, the group of all real $n \times n$ nonsingular matrices), $\mu$ the Haar measure on $G$, and $A$ the algebra of all bounded, integrable functions on $G$, with multiplication defined as convolution. The pair $(A, \mu)$ provides an example of the sort described in the beginning.

Let $H=L_{2}(G, \mu)$ where $G$ is the group of all transformations $c x+d, c, d$ rational, and $\mu$ is the counting measure on $G$. For $f, g$ in $H$, and $a, b$ in $G$, let $U_{a}$ be defined thus: $U_{a} f=g$, where $g(b)=f(b a)$. Let $M$ be the ring of all bounded operators which commute with $U_{a}$ for all $a$. Every bounded operator $A$ in $H$ is representable in the form of a bounded numerical matrix $A \sim\left\|\eta_{a, b}\right\|, a, b$ in $G$. For any $A$ in $M$, set $\tau(A)=\eta_{e, e}(e$ being the identity of $G)$. Let $I$ denote the identity operator. For arbitrary $C, D$ in $M, \tau(C D)=\tau(D C)$, and $\tau(I)=1 . M$, a factor of type $\mathrm{II}_{1}$, is the noncommutative analogue of bounded, complex random variables on a nonatomic probability space.

A special case of a type $\mathrm{II}_{1}$ factor, bearing the appellation "approximately finite factor," arises in a natural way, in the theory of Fermi-Dirac quantization as described in the papers [7], [8], and [9].

The integration theory, developed by Segal in the most general setting, may be epitomized thus. A ring of operators, with a trace defined on some elements thereof is given. The trace is then extended to a wider class via suitable convergence concepts. And, for this enlarged ensemble (whose elements are called integrable) analogues of standard measure-theoretic results are obtained.

In an arbitrary gage space, Segal introduced, and made a fairly extensive study of, the concepts of measurable operators, convergence nearly everywhere, and

Received by the editors September 27, 1966. 
integrable and square-integrable operators, besides obtaining extensions for such basic results as the Riesz-Fischer, Radon-Nikodym and Lebesgue monotone convergence theorems and for a reformulation of the Fubini Theorem. Thus a development of the theory of rings, collateral to measure theory was rendered possible by Segal's work.

The kind of work initiated by Segal was further pursued by Stinespring [10]. He defined convergence in measure in gage spaces, studied the interrelation between convergence in measure and convergence nearly everywhere, and proved, inter alia, noncommutative versions of Fatou's Lemma and Fubini's Theorem. Deserving of special mention are his results which state that under some mild restrictions, a continuous function preserves convergence in the $L_{2}$-mean and that a sequence $\left\{T_{n}\right\}$ of selfadjoint measurable operators converges in measure to a selfadjoint measurable operator $T$, if and only if, for any real continuous function $\Phi$ with compact support on the real line $\left\{\Phi\left(T_{n}\right)\right\}$ converges in measure to $\Phi(T)$.

Drawing freely on the works of Segal and Stinespring, we have, in the present paper, extended certain standard results in probability theory to finite rings.

1. Summary and preliminaries. Throughout this paper, the notation and terminology will be the same as those of [6] and [10]. Let $(H, \alpha, m)$ be a gage space in the sense of [6], $I$ the identity operator and $m$, a gage on $\alpha$ with $m(I)=1$, and which is regular, i.e., for any projection $P, m(P)=0$ implies $P=0$. By an operator we shall always mean an operator measurable with respect to (w.r.t.) $\alpha$ is the sense of [6].

This paper is divided into several sections.

In $\$ 2$, we prove that convergence in measure is preserved by a continuous function which is expressible as the sum of a finite number of monotonic continuous functions. We indicate a few applications of this result including one to operatorentropy.

In §3, we prove a noncommutative version of Egoroff's Theorem and its converse. We then obtain a necessary and sufficient condition for convergence in measure and convergence nearly everywhere to coincide.

In $\$ 4$, we introduce the notion of the distribution function of a selfadjoint measurable operator with respect to a faithful state (positive, normal, linear functional) $\phi$ of $\alpha$. Let $F_{n}$ be the distribution function of $T_{n}, n=1,2, \ldots$, and $F$ that of $T$, with respect to $\phi$. Let $x$ be any continuity-point of $F$ (i.e., a point at which $F$ is continuous). We prove that if $\left\{T_{n}\right\}$ converges in measure to $T$, then $F_{n}(x) \rightarrow F(x)$. Let $\phi=m$, and let $F_{n}, F$, and $x$ be as above. Then we also show that $\left\{T_{n}\right\}$ converges to $T$ in measure if and only if, for each such $x,\left\{P_{n}^{x}\right\}$ converges in measure to $P^{x}$, where $P_{n}^{x}$ and $P^{x}$ are spectral projections of $T_{n}$ and $T$ respectively corresponding to the infinite closed interval $(-\infty, x]$. We then deduce a few simple corollaries.

In $\S 5$, we prove two dominated convergence theorems, which, in the case of a finite gage space, are stronger than the corresponding results of Stinespring. As 
applications, we show, in $\S 6$, that if $\left\{T_{n}\right\}$ is a sequence of nonnegative squareintegrable operators converging in the $L_{2}$-mean to an operator $T$, then the operatorentropy of $T_{n}$ tends in the $L_{1}$-mean to that of $T$, and for any bounded selfadjoint operator $R$, the information about $T_{n}$ contained in $R$ (as defined by Umegaki and Nakamura) tends to the information about $T$ contained in $R$.

Although the notation and terminology of this paper will be the same as those of the papers [6] and [10], we shall define some concepts and explain some symbols which we shall repeatedly deal with.

Let $(H, \alpha, m)$ be the underlying gage space. For any operator $A$ in $\alpha,\|A\|$ will denote the operator norm of $A$. For any measurable operator $T$ (not necessarily bounded), $|T|$ will denote $\left(T^{*} T\right)^{1 / 2}$. The extension of the gage $m$ to the class of all integrable operators will also be denoted by $m$. The $L_{1}$-norm of $T$, denoted by $\|T\|_{1}$, is the number $m(|T|)$. The $L_{2}$-norm of $T$, denoted by $\|T\|_{2}$, is the number $\left[m\left(T^{*} T\right)\right]^{1 / 2}$. For any two projections $P$ and $Q$ in $\alpha, P \vee Q$ and $P \wedge Q$ will denote respectively the lattice-sum and lattice-product of $P$ and $Q$. A projection and its range will be denoted by the same symbol. For any two measurable operators $A$ and $B, A+B$ and $A \cdot B$ will denote respectively the strong-sum and strong-product of $A$ and $B$.

A sequence $\left\{T_{n}\right\}$ will be said to converge in measure to $T$, if given any $\varepsilon>0$, there exists a sequence $\left\{Q_{n}\right\}$ of projections in $\alpha$, such that $\left\|\left(T_{n}-T\right) Q_{n}\right\|<\varepsilon$ for all $n$ and $m\left(Q_{n}\right) \rightarrow 1$.

It will be said to converge nearly everywhere to $T$ if, given any $\varepsilon>0$, there exists a sequence $\left\{Q_{n}\right\}$ of projections in $\alpha$, such that $\left\|\left(T_{n}-T\right) Q_{n}\right\|<\varepsilon$ for all $n$ and $Q_{n} \uparrow I$.

It will be said to converge to $T$ in the $L_{p}$-mean if $\left\|T_{n}-T\right\|_{p} \rightarrow 0$, as $n \rightarrow \infty$, $p=1,2$, and converge to $T$ almost uniformly if, given any $\delta>0$, one can find a projection $P$ in $\alpha$ with $m(P) \geqq 1-\delta$ and such that

$$
\left\|\left(T_{n}-T\right) P\right\| \rightarrow 0 \text { as } n \rightarrow \infty \text {. }
$$

The following results of Stinespring, which we shall refer to as $\left[D_{1}\right],\left[D_{2}\right]$, and $\left[D_{3}\right]$ respectively, will be frequently used in this paper.

$\left[\mathrm{D}_{1}\right]$ [10, p. 32, Corollary 5.2]. "A sequence $\left\{A_{n}\right\}$ of measurable operators converges in measure to a measurable operator $A$ if and only if, for any $\varepsilon>0, m\left(R_{n}^{\varepsilon}\right) \rightarrow 0$ as $n \rightarrow \infty$, where $R_{n}^{\varepsilon}$ is the spectral projection of $\left|A_{n}-A\right|$ corresponding to $(\varepsilon, \infty)$."

$\left[\mathrm{D}_{2}\right]\left[10\right.$, p. 33, Theorem 5.5]. "A sequence $\left\{T_{n}\right\}$ of selfadjoint measurable operators converges in measure to a selfadjoint measurable operator $T$ if and only if, for any continuous function $\sigma$ with compact support on the real line, $\sigma\left(T_{n}\right) \rightarrow \sigma(T)$ in the $L_{2}$-mean."

$\left[\mathrm{D}_{3}\right][10, \mathrm{p} .24]$. "Convergence in the $L_{p}$-mean implies convergence in measure," $p=1,2$.

\section{Preservation of convergence in measure by continuous functions.}

THEOREM 2.1. Let $\left\{T_{n}\right\}$ be a sequence of operators converging in measure to an operator $T$. Let $R_{n}^{\varepsilon}$ denote the spectral projection of $\left|T_{n}-T\right|$ corresponding to the interval $(\varepsilon, \infty)$. Then there exists a subsequence $\left\{n_{k}\right\}$ such that $\sum_{k=1}^{\infty} m\left(R_{n_{k}}^{\varepsilon}\right)<\infty$. 
Proof. In the case of an arbitrary gage space, it has been proved by Stinespring $\left[10\right.$, p. 23] that $\left\{T_{n}\right\}$ has a subsequence which converges nearly everywhere. His methods together with the finiteness of the gage yield the desired result.

THEOREM 2.2. Let $\left\{T_{n}\right\}$ be a sequence of selfadjoint operators converging in measure to a selfadjoint operator $T$. Let $\Phi$ be a real-valued continuous function, expressible as the sum of a finite number of real and monotonic continuous functions. Then $\left\{\Phi\left(T_{n}\right)\right\}$ converges in measure to $\Phi(T)$.

Proof. Case 1. $\Phi$ is strictly increasing and continuous, and its range is the whole real line.

Proof. Let $\Phi\left(T_{n}\right)=S_{n}$ and $\Phi(T)=S$. Then $S$ and $S_{n}$ are selfadjoint. Let " $a$ " be any continuous function with compact support. Let $a \cdot \Phi$ denote the composite map defined thus: For any real number $\lambda,(a \cdot \Phi)(\lambda)=a(\Phi(\lambda))$. It is easy to verify that $(a \cdot \Phi)\left(T_{n}\right)=a\left(\Phi\left(T_{n}\right)\right)=a\left(S_{n}\right)$. Since $\Phi$ is strictly increasing and its range is the whole real line, $a \cdot \Phi$ is once again a continuous function with compact support. Since $\left\{T_{n}\right\}$ converges in measure to $T$, it follows by $\left[\mathrm{D}_{2}\right]$ that $\left\{(a \cdot \Phi)\left(T_{n}\right)\right\}$ converges in the $L_{2}$-mean to $(a \cdot \Phi)(T)$, i.e., $\left\{a\left(S_{n}\right)\right\}$ converges in the $L_{2}$-mean to $a(S)$. As " $a$ " is arbitrary, by applying $\left[\mathrm{D}_{2}\right]$ again, it follows that $\left\{S_{n}\right\}$ converges in measure to $S$.

Case 2. $\Phi$ is continuous and strictly increasing, and its range is a bounded interval.

Proof. Let $a(\lambda)=\lambda$. Let $b(\lambda)=a(\lambda)+\Phi(\lambda)$. Then, by case $1,\left\{b\left(T_{n}\right)\right\}$ converges in measure to $b(T)$, so that $\left\{b\left(T_{n}\right)-T_{n}\right\}$ converges in measure to $b(T)-T$, i.e., $\left\{\Phi\left(T_{n}\right)\right\}$ converges in measure to $\Phi(T)$.

Case 3. $\Phi$ is strictly increasing and continuous, and its range is an unbounded interval with a finite left-hand endpoint.

Proof. Let $\Phi(0)=k$. Without loss of generality, we may assume $k=0$, as otherwise we may consider $f(\lambda)=\Phi(\lambda)-k$. Define a new function $g(\lambda)$ thus: For $\lambda>0$, $g(\lambda)=\Phi(\lambda)$. For $\lambda=0$, and $\lambda<0, g(\lambda)=\lambda$. Let $b(\lambda)=g(\lambda)+\Phi(\lambda)$. By case $1,\left\{g\left(T_{n}\right)\right\}$ converges in measure to $g(T)$ and $\left\{b\left(T_{n}\right)\right\}$ to $b(T)$. Hence $\left\{\Phi\left(T_{n}\right)\right\}$ converges in measure to $\Phi(T)$. The case where the range of $\Phi$ is an interval bounded on the right, but not on the left, can be disposed of similarly. Thus the Theorem has been proved for any strictly increasing continuous function $\Phi$. Now let $\Phi$ be continuous and increasing (but not necessarily strictly). Let $g(\lambda)=\Phi(\lambda)+\lambda$. Then, since $g$ is strictly increasing $\left\{g\left(T_{n}\right)\right\}$ converges in measure to $g(T)$, so that $\left\{\Phi\left(T_{n}\right)\right\}=\left\{g\left(T_{n}\right)-T_{n}\right\}$ converges in measure to $g(T)-T=\Phi(T)$. Similarly the theorem can be proved when $\Phi$ is decreasing and continuous. Hence the theorem is true when $\Phi$ is a finite linear combination of monotonic continuous functions.

Applications. 1. Let $S$ be a selfadjoint operator, $P$ the spectral projection of $S$ corresponding to $[0, \infty)$, and $Q=I-P$. The operator $S P$ will be denoted by $S^{+}$ and the operator $-S Q$ by $S^{-}$. Let $\left\{T_{n}\right\}$ converge in measure to $T$. Then one can show that $\left\{T_{n}^{+}\right\}$converges in measure to $T^{+}$and $\left\{T_{n}^{-}\right\}$in measure to $T^{-}$.

Proof. Let $g(\lambda)=\lambda(\lambda \geqq 0)$ and $=0(\lambda<0)$. 
Then, by the above theorem, $\left\{g\left(T_{n}\right)\right\}$ converges in measure to $g(T)$. But $g\left(T_{n}\right)=T_{n}^{+}$ and $g(T)=T^{+}$. The other part can be proved similarly.

2. Let $\left\{T_{n}\right\}$ be an arbitrary sequence of operators (not necessarily selfadjoint) converging in measure to $T$. Then $\left\{\left|T_{n}\right|\right\}$ converges in measure to $|T|$.

Proof. It is known [10, pp. 28 and 32] that if $\left\{T_{n}\right\}$ converges in measure to $T$, then $\left\{T_{n}{ }^{*} T_{n}\right\}$ converges in measure to $T^{*} T$. Let $g(\cdot)$ be defined thus: $g(\lambda)=0$, $\lambda \leqq 0$, and $g(\lambda)=+(\lambda)^{1 / 2}$, for $\lambda \geqq 0$. Let $S_{n}=T_{n}{ }^{*} T$ and $S=T^{*} T$. Then $\left\{g\left(S_{n}\right)\right\}$ converges in measure to $g(S)$.

3. Let $\left\{T_{n}\right\}$ be a sequence of nonnegative operators. For each $n,-T_{n} \log T_{n}$ is called the operator entropy of $T_{n}$, and $m\left(-T_{n} \log T_{n}\right)$ is called the numerical entropy of $T_{n}$. Let $\left\{T_{n}\right\}$ converge in measure to $T$. Then $T$ can be shown to be nonnegative so that $T \log T$ can be defined. Now the function $\lambda \log \lambda$ is expressible as the sum of a finite number of monotonic, continuous functions so that, by the above theorem, the operator-entropy of $T_{n}$ converges in measure to the operatorentropy of $T$. Let now $\left\{T_{n}\right\}$ be uniformly bounded, i.e., there exists a positive integer $k$ such that $\left\|T_{n}\right\| \leqq k$ for all $n$. In this case, there is convergence even in the $L_{1}$-mean. Hence $m\left(-T_{n} \log T_{n}\right) \rightarrow m(-T \log T)$. In other words, the entropy function is continuous in bounded sets of $\alpha$ in the topology of convergence in the $L_{1}$-mean.

4. Let $g$ be a strictly monotonic and real function, continuous everywhere on the real line. Let $g(0)=0$. Also let $g$ be bounded, i.e., for some positive integer $k$, $|g(\lambda)|<k$ for all real $\lambda$. For any two measurable operators $T$ and $S$, define $p(T, S)=$ $m(g(|T-S|))$. Then $\left\{T_{n}\right\}$ converges in measure to $T$ if and only if $p\left(T_{n}, T\right) \rightarrow 0$ as $n \rightarrow \infty$.

Proof. First let $\left\{T_{n}\right\}$ converge in measure to $T$. This implies that $\left\{\left|T_{n}-T\right|\right\}$ converges in measure to zero. Let $S_{n}=\left|T_{n}-T\right|$. As $\left\{S_{n}\right\}$ converges in measure to zero, it follows by Theorem 2 that $\left\{g\left(S_{n}\right)\right\}$ converges in measure to $g(0)=0$. Since $\left\|g\left(S_{n}\right)\right\| \leqq k$ for all $n$, it even follows that $m\left(g\left(S_{n}\right)\right) \rightarrow 0$, i.e., $p\left(T_{n}, T\right) \rightarrow 0$.

Conversely, let $p\left(T_{n}, T\right) \rightarrow 0$. This implies that $m\left(g\left(S_{n}\right)\right) \rightarrow 0$. As $g\left(S_{n}\right)$ is nonnegative for each $n$, it follows that $\left\{g\left(S_{n}\right)\right\}$ converges to zero in measure. Now, if possible, let $\left\{S_{n}\right\}$ not converge in measure to zero. By $\left[D_{1}\right]$ there exists therefore some $\varepsilon>0$ such that the sequence $\left\{m\left(R_{n}^{\varepsilon}\right)\right\}$ does not converge to zero, where $R_{n}^{\varepsilon}$ is the spectral projection of $S_{n}$ corresponding to $(\varepsilon, \infty)$. Hence there exists at least one subsequence, denoted by $\left\{m\left(R_{n_{k}}^{\varepsilon}\right)\right\}$, which converges to a strictly positive number $L$. As $g$ is strictly monotonic, $g(\varepsilon)>g(0)=0$. Now

$$
\begin{aligned}
0<g(\varepsilon) L & =g(\varepsilon) \underset{n_{k} \rightarrow \infty}{\mathrm{Lt}} m\left(R_{n_{k}}^{\varepsilon}\right) \\
& =\underset{n_{k} \rightarrow \infty}{\mathrm{Lt}} m\left(g(\varepsilon) \cdot R_{n_{k}}^{\varepsilon}\right) \\
& <\underset{n_{k} \rightarrow \infty}{\mathrm{Lt}} m\left(g\left(S_{n}\right)\right) \\
& =0 .
\end{aligned}
$$

Thus $L=0$. And this contradiction shows that $\left\{S_{n}\right\}$ converges in measure to zero. 


\section{Egoroff's Theorem and the various modes of convergence.}

THEOREM 3.1. A sequence $\left\{T_{n}\right\}$ converges nearly everywhere to $T$ if and only if it converges to $T$ almost uniformly.

REMARK. This theorem generalizes Egoroff's Theorem and its converse.

Proof. Let $\left\{T_{n}\right\}$ converge to $T$ nearly everywhere. We shall show that it converges to $T$ almost uniformly. Without loss of generality we may take $T=0$. Let $\left\{\varepsilon_{k}\right\}$ be a sequence of positive numbers converging to zero. By the definition of nearly everywhere convergence, corresponding to $\varepsilon_{j}(j=1,2, \ldots)$ there exists a sequence $\left\{Q_{j n}\right\}$ such that $\left\|T_{n} Q_{j n}\right\|<\varepsilon_{j}$ for all $n$ and for a fixed $j, Q_{j n} \uparrow I$, as $n \rightarrow \infty$. Given any positive number $\delta>0$, one can, in view of the latter property, find a projection $Q_{1 n_{1}}$ such that $m\left(Q_{1 n_{1}}\right)>1-\delta / 2$ and similarly a projection $Q_{2} n_{2}$ such that $m\left(Q_{2 n_{2}}\right)>1-\delta / 2^{2}, \ldots$ and in general a projection $Q_{j n_{j}}$ (from the sequence $\left\{Q_{j n}\right\}$ ), such that $m\left(Q_{i n_{j}}\right)$ $>1-\delta / 2^{j}$. For convenience, write $Q_{j}=Q_{j, n_{j}}$. Let $S=\bigwedge_{j=1}^{\infty} Q_{j}$. Let $S^{\perp}=I-S$. Then $m\left(S^{\perp}\right)=m\left(\bigvee_{j=1}^{\infty} Q_{j}^{\perp}\right) \leqq \sum_{j} m\left(Q_{j}^{\perp}\right)=\sum_{j} \delta / 2^{j}=\delta$. Hence $m(S) \geqq 1-\delta$. Let an arbitrary positive number $\varepsilon$ be given. Since $\varepsilon_{k} \rightarrow 0$ as $k \rightarrow \infty, \varepsilon_{k}<\varepsilon$ for all $k \geqq$ some positive integer $L$. Hence for any $k \geqq n_{L},\left\|T_{k} S\right\| \leqq\left\|T_{k} Q_{L, n_{L}}\right\| \leqq \varepsilon_{L}<\varepsilon$. And $m(S)>1-\delta$.

This being true of any arbitrary $\delta>0$, it follows that $\left\{T_{n}\right\}$ converges to 0 nearly everywhere.

CONVERSE. Let $\left\{T_{n}\right\}$ converge to 0 almost uniformly. We shall show that $\left\{T_{n}\right\}$ converges to 0 nearly everywhere.

Proof. Let $\delta$ be a positive number less than 1 . In view of our assumption, there exists a sequence $\left\{S_{k}\right\}$ of projections such that $m\left(S_{k}\right)>1-\delta / 2^{k}$ and for each fixed $k$, $\left\|T_{n} S_{k}\right\| \rightarrow 0$ as $n \rightarrow \infty$. Let $R_{1}=\bigwedge_{k=1}^{\infty} S_{k}, R_{2}=\bigwedge_{k=2}^{\infty} S_{k}, \ldots, R_{n}=\bigwedge_{k=n}^{\infty} S_{k}, \ldots$ Then $m\left(R_{n}^{\perp}\right) \leqq \sum_{j=n}^{\infty} \delta / 2^{j}$. So $m\left(R_{n}\right) \rightarrow 1$. As $m$ is regular, and $R_{k}<R_{k+1}$ for each $k$, it follows that $R_{n} \uparrow I$. Now for each $k, R_{k}<S_{k}$. Since for each fixed $k,\left\|T_{n} S_{k}\right\| \rightarrow 0$ as $n \rightarrow \infty$, given $k$, and $\varepsilon>0$, one can find a positive integer $N_{k}$ such that $\left\|T_{n} S_{k}\right\|<\varepsilon$ for all $n \geqq N_{k}(k=1,2,3, \ldots)$. Define a sequence $\left\{E_{k}\right\}$ of projections thus:

$$
\begin{aligned}
E_{1} & =E_{2}=\cdots=E_{N_{1}-1}=0 \text { (the zero projection), } \\
E_{N_{1}} & =E_{N_{1}+1}=\cdots=E_{N_{1}+N_{2}-1}=R_{1}, \\
E_{N_{1}+N_{2}} & =E_{N_{1}+N_{2}+1}=\cdots=E_{N_{1}+N_{2}+N_{3}-1}=R_{2} .
\end{aligned}
$$

And, in general,

$$
E_{N_{1}+N_{2}+\cdots+N_{k}}=\cdots=E_{N_{1}+N_{2}+\cdots+N_{k+1}-1}=R_{k}, \ldots, \quad k=1,2, \ldots,
$$

The sequence $E_{n} \uparrow I$. For $n<N_{1},\left\|T_{n} E_{n}\right\|=0$ and so is less than $\varepsilon$. For $n \geqq N_{1}, n$ lies between two integers $N_{1}+\cdots+N_{k}$ and $N_{1}+N_{2}+\cdots+N_{k+1}-1$ so that $\left\|T_{n} E_{n}\right\|=\left\|T_{n} R_{k}\right\|<\left\|T_{n} S_{k}\right\|<\varepsilon$ (since $R_{k} \leqq S_{k}$ ). Thus for all $n,\left\|T_{n} E_{n}\right\|<\varepsilon$. For any given $\varepsilon>0$, the choice of one such sequence $\left\{E_{n}\right\}$ being possible, it follows that $\left\{T_{n}\right\}$ converges nearly everywhere to 0 . Hence the theorem is proved.

In what follows a projection $P$ in $\alpha$ will be said to be minimal if, for any projection $Q$ in $\alpha, Q \leqq P$ implies $Q=P$ or $Q=0$. 
THEOREM 3.2. Convergence in measure and convergence nearly everywhere are equivalent if and only if each projection in $\alpha$ contains a minimal projection.

Proof. Let each projection contain a minimal projection. We shall show that these two notions of convergence coincide. Let $I=P_{1}+P_{2}+\cdots+P_{n}+\cdots$ be some resolution of the identity into a sequence of pairwise orthogonal minimal projections. Let $m\left(P_{i}\right)=\delta_{i}$. As $\delta_{i} \geqq 0$, and $\sum_{i=1}^{\infty} \delta_{i}=1$, we can assume without loss of generality that $\delta_{1} \geqq \delta_{2} \geqq \cdots \geqq \delta_{n} \geqq \cdots$. Let $\left\{T_{n}\right\}$ be a sequence of operators converging in measure to an operator $T$. We may take $T=0$. By the definition of convergence in measure, given $\varepsilon$ there exists a sequence $\left\{Q_{n}\right\}$ of projections such that $m\left(Q_{n}\right) \rightarrow 1$ and $\left\|T_{n} Q_{n}\right\|<\varepsilon$ for all $n$. As $m\left(Q_{n}\right) \rightarrow 1$, there exists a positive integer $N_{1}$ such that $m\left(Q_{n}\right)>1-\delta_{1} / 2$ for all $n \geqq N_{1}$, and similarly a positive integer $N_{2}$ such that $m\left(Q_{n}\right)>1-\delta_{1} / 2-\delta_{2} / 2$ for all $n \geqq N_{2}, \ldots$, and in general a positive integer $N_{k}$ such that $m\left(Q_{n}\right)>1-\delta_{1} / 2-\delta_{2} / 2 \cdots-\delta_{k} / 2$ for all $n \geqq N_{k}, k$ ranging over all positive integral values. Obviously for $n \geqq N_{1}, Q_{n} \wedge P_{1}$ is nonnull. But as $P_{1}$ is minimal, this implies $P_{1} \leqq Q_{n}$ for $n \geqq N_{1}$. Similarly $P_{1} \vee P_{2} \leqq Q_{n}$ for $n \geqq N_{1}+N_{2}$ etc.

$$
\begin{aligned}
R_{1} & =0=\cdots=R_{N_{1}-1}, \\
R_{N_{1}} & =P_{1}=\cdots=R_{N_{1}+N_{2}-1}, \\
R_{N_{1}+N_{2}} & =P_{1} P_{2}=\cdots=R_{N_{1}+N_{2}+N_{3}-1}, \text { etc. }
\end{aligned}
$$

As $\sum_{i=1}^{\infty} m\left(P_{i}\right)=1$, it follows that $R_{n} \uparrow I$ and $\left\|T_{n} R_{n}\right\|<\varepsilon$ for all $n$. Hence convergence in measure implies convergence nearly everywhere. Since, in the case of a finite gage space, convergence in measure is always implied by convergence nearly everywhere, it follows that they are equivalent in this case.

CONVERSE. Let convergence in measure and convergence nearly everywhere coincide. We shall show that each projection has to contain a minimal projection. If not, let there exist a nonnull projection $P$, with the following property: $m(P)=\delta$ and for any $\beta$ with $0<\beta<\delta$, there exists a projection $R<P$, with $m(R)=\beta$. We can show that this implies that for any $n, P$ can be expressed as the sum of $n$ pairwise orthogonal projections $P_{1 n}, \ldots, P_{n n}$, such that $m\left(P_{i n}\right)=m(P) / n, i=1,2, \ldots, n$. Now clearly the sequence $P_{11}, 2 P_{21}, 2 P_{22}, 3 P_{31}, 3 P_{32}, 3 P_{33}, \ldots, n P_{n 1}, n P_{n 2}, \ldots, n P_{n n}, \ldots$ converges in measure to 0 . If possible, let this converge nearly everywhere to 0 . By the noncommutative version of Egoroff's Theorem, one can find a projection $S$ with $m(S)>1-m(P)$ and such that $\left\|n P_{n i} S\right\| \rightarrow 0$. Let $\varepsilon<1$. Hence there exists a positive integer $N$ such that $\left\|n P_{n i} S\right\|<\varepsilon \cdots(1)$; for all $n \geqq N . P \wedge S$ is nonnull, as $m(S)>1-m(P)$. Let $x$ be a unit vector in $P \wedge S$. Then for any $n \geqq N, 1=\|x\|^{2}$ $=\|P x\|^{2}=\left\|P_{n 1} x\right\|^{2}+\cdots+\left\|P_{n n} x\right\|^{2}$.

But by (1), $\left\|P_{n i} \cdot r\right\|^{2}<\varepsilon^{2} / n^{2}, i=1,2, \ldots, n$. Hence

$$
1<\varepsilon^{2} / n^{2}+\cdots+\varepsilon^{2} / n^{2}(n \text { times })=\varepsilon^{2} / n<\varepsilon^{2}<1 .
$$

This contradiction proves the result.

4. Weak convergence. Let $(X, \Gamma, P)$ be a probability space. By a random variable is meant a real, almost everywhere finite-valued $\Gamma$-measurable function. 
With each random variable $\xi$, one can associate a probability measure $\mu$ defined on the Borel sets of line as $\mu(E)=P\left[\xi^{-1}(E)\right]$. This $\mu$ is known as the probability measure corresponding to $\xi$. Let $(-\infty, x]=\{y:-\infty<y \leqq x\}$. The function $F$ defined by $F(x)=\mu\{(-\infty, x]\}$ is called the distribution function of $\xi . F$ is always right-continuous. A point $x$ at which $F$ is also left-continuous is known as a continuity-point of $F$. It can easily be seen that $x$ is a continuity-point of $F$ if and only if $\mu(x)=0$. The set of continuity-points of a distribution function is known to be dense on the real line.

Now let $T$ be any selfadjoint operator and $P_{E}$ its spectral projection corresponding to the Borel set $E$. We shall call the measure $\mu$, defined by $\mu(E)=m\left(P_{E}\right)$, the probability measure associated with $T$. And the point function $F$, defined at any point $x$ by $F(x)=\mu\{(-\infty, x]\}$, we shall call the distribution function of $T$. Following the measure-theoretic case, we define that a sequence $\left\{T_{n}\right\}$ of selfadjoint operators with distribution functions $\left\{F_{n}\right\}$ converges weakly to a selfadjoint operator $T$ with distribution function $F$ if, at every continuity point $x$ of $F, F_{n}(x) \rightarrow F(x)$. (As before, if $\mu_{n}$ is the probability measure associated with $T_{n}, n=1,2, \ldots$, and $\mu$ is the measure associated with $T$, then it is known that a necessary and sufficient condition for weak convergence [1, p. 33] is that $\int_{R} g d \mu_{n} \rightarrow \int_{R} g d \mu$, where $g$ is any bounded continuous function, and $R$ denotes the real line) or, what is the same, $m\left(g\left(T_{n}\right)\right) \rightarrow m(g(T))$.

More generally, let $\sigma$ be any faithful state of $\alpha$, with $\sigma(I)=1$. Let $T$ be any selfadjoint operator. Since $\sigma$ is completely additive, using $\sigma$ one can as before associate a distribution function $G$ with $T$. This $G$ we shall call the distribution function of $T$ with respect to $\sigma$. As $\sigma$ is faithful, it can easily be seen that a point $x$ is a continuity point of $G$ if and only if the spectral projection of $T$ corresponding to the singleton $x$ is the zero projection. When we say simply the distribution function of an operator, we mean its distribution function with respect to the gage $m$.

In what follows $\left\{T_{n}\right\}$ will denote a sequence of selfadjoint operators converging in measure to a selfadjoint operator $T, F$ the distribution function of $T$, and $F_{n}$ that of $T_{n}(n=1,2, \ldots)$. We shall now state and prove four theorems.

THEOREM 4.1. Let $x$ be an arbitrary continuity point of $F$. Then $F_{n}(x) \rightarrow F(x)$ as $n \rightarrow \infty$.

THEOREM 4.2. Let $\sigma$ be any faithful state. Let $G$ be the distribution function of $T$ with respect to $\sigma$, and $G_{n}$ that of $T_{n}$ with respect to $\sigma ; n=1,2, \ldots$ Let $x$ be an arbitrary continuity point of $G$. Then $G_{n}(x) \rightarrow G(x)$.

THEOREM 4.3. Let $\left\{A_{n}\right\}$ and $\left\{B_{n}\right\}$ be two sequences of selfadjoint operators such that $\left\{A_{n}-B_{n}\right\}$ converges in measure to zero. Let $\left\{A_{n}\right\}$ converge weakly to a selfadjoint operator $A$ (i.e., if $H_{n}$ is the distribution of $A_{n}$, and $H$ that of $A$, then $H_{n}(x) \rightarrow H(x)$ at every point $x$ which is a continuity point of $H)$. Then $\left\{B_{n}\right\}$ also converges weakly to $A$. 
THEOREM 4.4. Let $x$ be an arbitrary continuity point of $F$. Let $P_{n}^{x}$, for each $n$, denote the spectral projection of $T_{n}$ corresponding to the interval $(-\infty, x]$ and $P^{x}$ that of $T$ corresponding to the same interval. Then a necessary and sufficient condition for $\left\{T_{n}\right\}$ to converge in measure to $T$ is that, corresponding to each continuity point $x$ of $F,\left\{P_{n}^{x}\right\}$ converges in measure to $P^{x}$.

RemarKs. Theorems 4.1 and 4.2 are consequences of Theorem 4.4; they are stated separately, however, because the proof of Theorem 4.4 depends on that of Theorem 4.1. Theorem 4.2 is more general than Theorem 4.1 but cannot be proved directly and has only to be deduced from Theorem 4.4.

\section{Proof of Theorem 4.1.}

Case 1. Let $T_{n}$ 's be uniformly bounded, i.e., there exists a positive integer $k$ such that $\left\|T_{n}\right\|<k$ for all $n$. In this case, the proof is exceedingly simple. Clearly for each $n$, the spectrum of $T_{n}$ is contained in the closed interval $[-k, k]$. Let $\mu_{n}$ be the probability measure associated with $T_{n}$. It is easy to verify that all the $\mu_{n}$ 's vanish outside the compact set $[k, k]$. Hence, in this case, to establish weak convergence it suffices to consider continuous functions $g$ with compact support. Now, $\int_{R} g d \mu_{n}=m\left(g\left(T_{n}\right)\right)$ and $\int_{R} g d \mu=m(g(T))$. But by the result $\left[\mathrm{D}_{2}\right],\left\{g\left(T_{n}\right)\right\}$ converges in the $L_{2}$-mean to $g(T)$; and hence, in particular,

$$
m\left(g\left(T_{n}\right)\right) \rightarrow m(g(T)), \text { i.e., } \int_{R} g d \mu_{n} \rightarrow \int_{R} g d \mu .
$$

Hence we have weak convergence in this case.

Case 2 (General case). Let $\left\{T_{n}\right\}$ be an arbitrary sequence of selfadjoint operators converging in measure to a selfadjoint operator $T$. Let $F_{n}$ be the distribution function of $T_{n}$, and $F$ that of $T$. Let $x$ be an arbitrary continuity point of $F$. To prove $F_{n}(x) \rightarrow F(x)$. Corresponding to $(-\infty, x]$, let $P_{n}^{x}$ be the spectral projection of $T_{n}$, and $P^{x}$ that of $T$. Then $F_{n}(x)=m\left(P_{n}^{x}\right)$ and $F(x)=m\left(P^{x}\right)$.

REMARK. The proof in the measure-theoretic case, as given in standard textbooks on probability theory, such as [2] and [3, p. 168], does not directly extend to the noncommutative case. The argument given in those books is of the following type: Let $(X, \beta, P)$ be a probability space. Let $f_{n}$ and $f$ be two random variables, $c$ any fixed point on the real line, and $\varepsilon>0$. Let $A=f_{n}^{-1}\{(-\infty, c]\}$ and $B=f^{-1}\{(c+\varepsilon, \infty)\}$. Let $C=X-B$. Then,

$$
A=A(\overline{)} B+A(\overline{)} C .
$$

This is the crucial decomposition on which the proof in the measure-theoretic case hinges. In the general case, let $P_{n}$ be the spectral projection of $T_{n}$ corresponding to $(-\infty, x], P$ the spectral projection of $T$ corresponding to $(c+\varepsilon, \infty)$, and $Q=I-P$. The equality $P_{n}=P_{n} \wedge P+P_{n} \wedge Q$ is not in general valid, since the associative law does not in general hold for the lattice of projections. In other words, the crucial decomposition (1) does not generalize to the case of rings of operators. Hence, we shall furnish a proof which is different from the one in the measure-theoretic case. 
Let $x$ be any fixed continuity point of $F$. We shall show that $m\left(P^{x}\right)$ is the only limit point of the bounded sequence $\left\{m\left(P_{n}^{x}\right)\right\}$. (Note that for all $n, 0 \leqq m\left(P_{n}^{x}\right) \leqq 1$.) Let $L$ be a limit point of $\left\{m\left(P_{n}^{x}\right)\right\}$. Hence there exists a subsequence, also denoted by $\left\{m\left(P_{n}^{x}\right)\right\}$, which converges to $L$. If possible, let $L<m\left(P^{x}\right)$. So, for some $\delta>0$, one can write $L=m\left(P^{x}\right)-2 \delta$. As $x$ is a continuity point of $F$, one can find for some $\varepsilon>0$, a point $x-\varepsilon$ (to the left of $x$ ) such that $F(x)-F(x-\varepsilon)<\delta / 2$. (Note that if $P^{x-\varepsilon}$ denotes the spectral projection of $T$ corresponding to $(-\infty, x-\varepsilon]$, then $F(x-\varepsilon)=m\left(P^{x-\varepsilon}\right)$.) Hence $L<m\left(P^{x-\varepsilon}\right)$. Since $m\left(P_{n}^{x}\right) \rightarrow L$, it follows that there exists a positive integer $N_{1}$ such that for all $n \geqq N_{1}, m\left(P_{n}^{x}\right)<L+\delta / 4<m\left(P^{x}\right)-\delta$. Let $A_{n}^{x}=I-P_{n}^{x}$. It follows that for

$$
n \geqq N_{1}, \quad m\left(A_{n}^{x} \wedge P^{x-\varepsilon}\right) \geqq \delta .
$$

Also, as $\left\{T_{n}\right\}$ converges in measure to $T$ it follows, by a result mentioned ábove, that if $S_{n}$ denotes the spectral projection of $\left|T_{n}-T\right|$ corresponding to the interval $[0, \varepsilon / 2)$, then $m\left(S_{n}\right) \rightarrow 1$ as $n \rightarrow \infty$. So there exists a positive integer $N_{2}$ such that $m\left(S_{n}\right)>1-\delta / 2$ for all $n \geqq N_{2}$. Let $N=\max \left(N_{1}, N_{2}\right)$. Then, for any $n \geqq N, K_{n}=$ $S_{n} \wedge A_{n}^{x} \wedge P^{x-\varepsilon}$ is nonnull. Let $c_{n}$ be any unit vector in $K_{n}$. Since $c_{n}$ belongs to $K_{n}$ and so to $P^{x-\varepsilon},\left(T c_{n}, c_{n}\right) \leqq x-\varepsilon$. And since $c_{n}$ is in $A_{n}^{x}$, one has $\left(T_{n} c_{n}, c_{n}\right) \geqq x$. As $c_{n}$ belongs to $S_{n}$, one has $\left\|\left|T_{n}-T\right| c_{n}\right\| \leqq \varepsilon / 2$. Thus

$$
\begin{aligned}
\varepsilon / 2 \geqq\left\|\left|T_{n}-T\right| c_{n}\right\| & =\left\|\left(T_{n}-T\right) c_{n}\right\| \geqq\left|\left(\left(T_{n}-T\right) c_{n}, c_{n}\right)\right| \\
& =\left|\left(T_{n} c_{n}, c_{n}\right)-\left(T c_{n}, c_{n}\right)\right| \geqq x-(x-\varepsilon)=\varepsilon .
\end{aligned}
$$

This contradiction shows that the limit point $L$ cannot be less than $m\left(P^{x}\right)$. Similarly by using the fact that $F$ is right-continuous, one can show that the assumption that $L>m\left(P^{x}\right)$ will also lead to a contradiction. Thus $m\left(P^{x}\right)$ is the only limit point of the bounded sequence $\left\{m\left(P_{n}^{x}\right)\right\}$. Hence $m\left(P_{n}^{x}\right) \rightarrow m\left(P^{x}\right)$, i.e., $F_{n}(x) \rightarrow F(x)$. Hence the theorem is proved.

Corollary 4.1. Let $x_{1}$ and $x_{2}$ be two continuity points of $F\left(x_{1}<x_{2}\right.$. Let $R_{n}$ be the spectral projection of $T_{n}$ corresponding to the interval $\left(x_{1}, x_{2}\right]$ (open at $x_{1}$ and closed at $x_{2}$ ), and $R$ the spectral projection of $T$ corresponding to the same interval. Then $m\left(R_{n}\right) \rightarrow m(R)$.

The proof is easy and is omitted.

Theorem 4.2 cannot be proved in the same way as above since, unlike a gage, a state may not be subadditive, i.e., for any two arbitrary projections $P$ and $Q$, the inequality $\sigma(P \vee Q) \leqq \sigma(P)+\sigma(Q)$ is not in general valid. We shall deduce Theorem 4.2 from Theorem 4.4 after proving the latter.

Proof of Theorem 4.3. Theorem 4.3 is not a consequence of Theorem 4.1 since the notion of weak convergence is not additive even in the commutative case. However, a proof can be given along the following lines: Let $F_{n}$ be the distribution function of $A_{n}, G_{n}$ the distribution function of $B_{n}(n=1,2, \ldots)$ and $F$ that of $A$. Let $x$ be any continuity point of $F$. Corresponding to the interval $(-\infty, x]$, let $P_{n}$ be the spectral projection of $A_{n}, Q_{n}$ that of $B_{n}$, and $P$ that of $A$. By assumption 
$m\left(P_{n}\right) \rightarrow m(P)$. Let $L$ be a limit point of the sequence $\left\{m\left(Q_{n}\right)\right\}$. As such there exists a subsequence, also denoted by $\left\{m\left(Q_{n}\right)\right\}$, which converges to $L$. If possible, let $L<m(P)$. So, for some $\delta>0, L=m(P)-2 \delta$. As in the argument of Theorem 4.1, one can find a number $\varepsilon>0$ such that $x-\varepsilon$ is a continuity point of $F$, and such that if $R$ is the spectral projection of $A$ corresponding to $(-\infty, x-\varepsilon]$, then $m(R)>m(P)$ $-\delta / 2$, so that $L<m(R)-\delta$. As $m\left(Q_{n}\right) \rightarrow L$, one can find a positive integer $M$ such that for all $n \geqq M, m\left(Q_{n}\right)<L+\delta<m(R)-\delta / 2$. For each $n$, let $R_{n}$ be the spectral projection of $A_{n}$ corresponding to $(-\infty, x-\varepsilon$ ]. Since $x-\varepsilon$ is a continuity point of $F$, and $\left\{A_{n}\right\}$ converges weakly to $A$, it follows that $m\left(R_{n}\right) \rightarrow m(R)$. Hence one can find a positive integer $N_{1}$ such that $m\left(Q_{n}\right)<m\left(R_{n}\right)-\delta / 4$, for all $n \geqq N_{1}$. Let $S_{n}$ denote the spectral projection of $\left|A_{n}-B_{n}\right|$ corresponding to the interval $[0, \varepsilon / 2)$. Since $\left\{A_{n}-B_{n}\right\}$ converges in measure to zero, $m\left(S_{n}\right) \rightarrow 1$ and so is $\geqq 1-\delta / 8$ for all $n \geqq$ some positive integer $N_{2}$. Let $N=\max \left(N_{1}, N_{2}\right)$. Then for any $n \geqq N, S_{n} \wedge Q_{n}^{\perp} \wedge R_{n}$ is nonnull. Let $c_{n}$ be any unit vector in $S_{n} \wedge Q_{n}^{\perp} \wedge R_{n}$. Then as $c_{n}$ is in $S_{n}$, $\left\|\left|A_{n}-B_{n}\right| c_{n}\right\| \leqq \varepsilon / 2$. But as $c_{n}$ is in $Q_{n}^{\perp},\left(B_{n} c_{n}, c_{n}\right) \geqq x$. And as $c_{n}$ is $R_{n},\left(A_{n} c_{n}, c_{n}\right)$ $<x-\varepsilon$. Thus $\varepsilon / 2>\left\|\left(A_{n}-B_{n}\right) c_{n}\right\| \geqq\left|\left(A_{n} c_{n}, c_{n}\right)-\left(B_{n} c_{n}, c_{n}\right)\right|=|(x-\varepsilon)-x|=\varepsilon$. This contradiction shows that $L$ cannot be less than $m(P)$. Similarly one can show that $L$ cannot be greater than $m(P)$. Thus $m(P)$ is the only limit point of the bounded sequence $\left\{\left(m\left(Q_{n}\right)\right\}\right.$, which proves the theorem.

As a consequence of the theorem, we obtain the following corollary, which, in the commutative case, has been proved by Slutsky.

COROLlary 4.2. Let $F_{n}$, for each $n$, be the distribution function of a selfadjoint operator $S_{n}$, and let $F$ be that of a selfadjoint operator $S$. Let $\left\{S_{n}\right\}$ converge to $S$ weakly, and let $\left\{D_{n}\right\}$ be another sequence of selfadjoint operators converging in measure to $\mathrm{cI}$ (c some real number, and I the identity operator). Let $G_{n}$ be the distribution function of $S_{n}+D_{n}$ and $G$ that of $S+c I$. Let $x$ be any continuity point of $F$. Then $G_{n}(x+c) \rightarrow G(X+c)$.

The proof of this corollary rests on the following proposition:

"Let $N$ be any selfadjoint operator with distribution function $H$. Let $c$ and $I$ be as in the above theorem. Let $J$ be the distribution function of $N+c I$. Then, for any point $y$ on the real line, $H(y)=J(y+c)$."

The proof is as follows: For any Borel set $E$ on the line, let $E+c$ denote the set of points $x+c$, where $x$ is any point of $E$. Then one can verify that the spectral projection of $N$ corresponding to $E$, is the same as the spectral projection of $N+c I$ corresponding to $E+c$.

Proof of the corollary. Set $A_{n}=S_{n}+D_{n}-c I, B_{n}=S_{n}$. Then $\left\{A_{n}-B_{n}\right\}$ converges in measure to zero. Also $\left\{B_{n}\right\}$ converges weakly to $S$. Hence by Theorem $4.3\left\{A_{n}\right\}$ converges weakly to $S$. Let $H_{n}$ be the distribution function of $A_{n}$ and $G_{n}$ that of $A_{n}+c I$. Then $H_{n}(x) \rightarrow F(x)$ where $x$ is any continuity point of $F$. By the above proposition $H_{n}(x)=G_{n}(x+c)$ and $F(x)=G(x+c)$. Hence $G_{n}(x+c) \rightarrow G(x+c)$ which proves the corollary. 
Proof of Theorem 4.4. Let $\left\{T_{n}\right\}$ converge in measure to $T$. Let $x$ be any continuity point of the distribution function $F$ of $T$. We shall now show that $\left\{P_{n}^{x}\right\}$ converges in measure to $P^{x}$. Let $\psi(\cdot)$ be the characteristic function of the set $(-\infty, x]$. Then $P_{n}^{x}=\psi\left(T_{n}\right)$ and $P^{x}=\psi(T)$. We shall prove that $m\left(\left|\psi\left(T_{n}\right)-\psi(T)\right|\right) \rightarrow 0$ which will imply that $\left\{\psi\left(T_{n}\right)\right\}$ converges to $\psi(T)$ in the $L_{1}$-mean and hence in measure. Let an arbitrary positive number $\varepsilon$ be given. The continuity points of $F$ being dense on the line, one can find a sufficiently small $\delta(\delta>0)$ such that $x+\delta$ is a continuity point of $F$ and $F(x+\delta)-F(x)<\varepsilon \cdots(1)$. Let $R^{x}$ denote the spectral projection of $T$, corresponding to $(x, x+\delta]$. As $x$ and $x+\delta$ are both continuity points of $F$, it follows by Corollary 4.1 that $m\left(R_{n}^{x}\right) \rightarrow m\left(R^{x}\right)$. Hence one can find a positive integer $N_{1}$ such that $\left|m\left(R_{n}^{x}\right)-m\left(R^{x}\right)\right|<\varepsilon$ for $n \geqq N_{1}$, i.e., $m\left(R_{n}^{x}\right)<2 \varepsilon$ for,$n \geqq N_{1}$. Now let $\sigma$ be the continuous function defined thus:

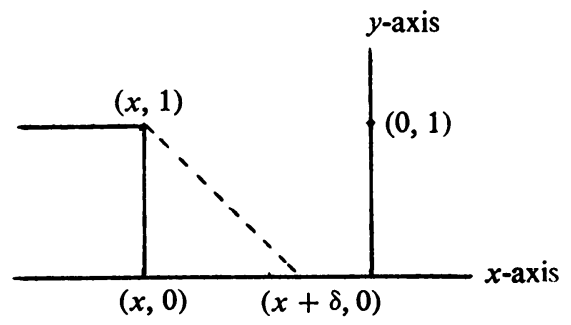

Graph of $\sigma$

For all $\lambda<x, \sigma(\lambda)=1$. For any $\lambda \geqq x+\delta, \sigma(\lambda)=0$. In the open interval $(x, x+\delta)$, the graph of $\sigma$ is the straight line joining the two points $(x, 1)$ and $(x+\delta, 0)$. Clearly, for any $\lambda,|\sigma(\lambda)-\psi(\lambda)|<1$, so that $\|\sigma(T)-\psi(T)\| \leqq 1$. Note that $\sigma(T)-\psi(T)$ is $\geqq 0$, so that

$$
\begin{aligned}
m(|\sigma(T)-\psi(T)|) & =m(\sigma(T)-\psi(T)) \\
& =m\left((\sigma(T)-\psi(T)) R^{x}\right) \leqq\|\sigma(T)-\psi(T)\| \cdot m\left(R^{x}\right)<\varepsilon .
\end{aligned}
$$

Similarly for $n>N_{1}$,

$$
\begin{aligned}
m\left(\left|\sigma\left(T_{n}\right)-\psi\left(T_{n}\right)\right|\right) & =m\left(\sigma\left(T_{n}\right)-\psi\left(T_{n}\right)\right) \\
& =m\left(\left(\sigma\left(T_{n}\right)-\psi\left(T_{n}\right)\right) \cdot R_{n}^{x}\right) \leqq\left\|\sigma\left(T_{n}\right)-\psi\left(T_{n}\right)\right\| \cdot m\left(R_{n}^{x}\right) \leqq m\left(R_{n}^{x}\right)<2 \varepsilon .
\end{aligned}
$$

As $\sigma$ is continuous and decreasing, it follows that $\left\{\sigma\left(T_{n}\right)\right\}$ converges in measure to $\sigma(T)$. Since this sequence is uniformly bounded, (bounded in norm by 1 ), convergence in measure implies convergence in the $L_{1}$-mean. So, given $\varepsilon$, there exists a positive integer $N_{2}$ such that $m\left(\left|\sigma\left(T_{n}\right)-\sigma(T)\right|\right)<\varepsilon$ for $n \geqq N_{2}$. Let $N=N_{1}+N_{2}$. Now for any $n \geqq N$ one has

$$
\begin{aligned}
m\left(\left|\psi(T)-\psi\left(T_{n}\right)\right|\right) & \leqq m(|\psi(T)-\sigma(T)|)+m\left(\left|\sigma(T)-\sigma\left(T_{n}\right)\right|\right)+m\left(\left|\sigma\left(T_{n}\right)-\psi\left(T_{n}\right)\right|\right) \\
& \leqq \varepsilon+\varepsilon+2 \varepsilon=4 \varepsilon,
\end{aligned}
$$

i.e., $m\left(\left|\psi\left(T_{n}\right)-\psi(T)\right|\right) \rightarrow 0$, i.e., $\left\{P_{n}^{x}\right\}$ converges to $P^{x}$ in the $L_{1}$-mean and hence in measure. And $x$ being any arbitrary continuity-point of $F$, the desired result follows. 
CONVERSE. For any continuity-point $x$ of $F$, let $P_{n}^{x}$ denote the spectral projection of $T_{n}$ and $P^{x}$ that of $T$, corresponding to $(-\infty, x]$. Let $\left\{P_{n}^{x}\right\}$ converge in measure to $P^{x}$. We shall now show that for any continuous function $\sigma$ with compact support on the real line, $\left\{\sigma\left(T_{n}\right)\right\}$ converges in the $L_{2}$-mean to $\sigma(T)$, and this will imply by the result $\left[\mathrm{D}_{2}\right]$ that $\left\{T_{n}\right\}$ converges in measure to $T$.

Let $\sigma$ have compact support $[-k, k]$. Hence $\sigma(-k)=0=\sigma(k)$. As $\sigma$ is uniformly continuous in $[-k, k]$, given any $\varepsilon>0$, one can find a positive number $\delta$, such that for any two points $x$ and $y$ in $[-k, k]$, one has $|\sigma(x)-\sigma(y)|<\varepsilon$, whenever $|x-y|<2 \delta$. Choose a point $x_{1}$ such that (1) $x_{1}>-k(2)$ the distance between $x_{1}$ and $-k$ exceeds $\delta / 2$ but is less than $\delta$ and (3) $x_{1}$ is a continuity point of $F$. Choose now successively points $x_{2}, x_{3}, \ldots, x_{N}$ such that (4) $x_{2}<x_{3}<\cdots<x_{N-1}<k$ and $x_{N}>k(5) \delta \geqq\left|x_{i+1}-x_{1}\right| \geqq \delta / 2$ and (6) each $x_{i}$ is a continuity point of $F, i=1,2, \ldots$, $N-1$. The choice of such a finite sequence $x_{1}, x_{2}, \ldots, x_{N}$ is possible as the continuity points of $F$ are dense on the real line.

Define a new function $\psi(\lambda)$ thus:

$$
\begin{aligned}
& \psi(\lambda)=0 \text { for } \quad \lambda \leqq x_{1}, \\
& \psi(\lambda)=\sigma\left(x_{1}\right), \quad x_{1}<A \leqq x_{2}, \\
& \psi(\lambda)=\sigma\left(x_{2}\right), \quad x_{2}<\lambda \leqq x_{3}, \quad \text { and } \\
& \psi(\lambda)=\sigma\left(x_{N-1}\right), \quad x_{N-1}<\lambda \leqq x_{N}, \\
& \psi(\lambda)=0, \quad \lambda>x_{N} .
\end{aligned}
$$

Clearly for any $\lambda$,

$$
|\psi(\lambda)-\sigma(\lambda)| \leqq \varepsilon .
$$

Corresponding to $\left(x_{i-1}, x_{i}\right]$, let $R_{n}^{i}$ denote the spectral projection of $T_{n}$ and $R^{i}$ that of $T(i=2, \ldots, N)$. By assumption, $\left\{R_{n}^{i}\right\}$ converges in measure to $R^{i}(i=1,2, \ldots, N)$, so that $\psi\left(T_{k}\right)=\sum_{i=2}^{N} \sigma\left(\lambda_{i-1}\right) R_{k}^{i}$ converges in measure to $\sum_{i=2}^{N} \sigma\left(\lambda_{i-1}\right) R^{i}=\psi(T)$. It is easy to verify that $|\psi(\lambda)-\sigma(\lambda)|<\varepsilon$ for all $\lambda$, so that $\left\|\psi\left(T_{n}\right)-\sigma\left(T_{n}\right)\right\|<\varepsilon$ for all $n$. Since $\left\{\psi\left(T_{n}\right)\right\}$ converges in measure to $\psi(T)$ and is uniformly bounded in norm, it follows that $\psi\left(T_{n}\right) \rightarrow \psi(T)$ in the $L_{2}$-mean, i.e., $\left\|\psi\left(T_{n}\right)-\psi(T)\right\|_{2} \rightarrow 0$ as $n \rightarrow \infty$. As such, there exists a positive integer $N_{1}$ such that $\left\|\psi\left(T_{n}\right)-\psi(T)\right\|_{2}<\varepsilon$ for all $n \geqq N_{1}$. Again, for each $n,\left\|\psi\left(T_{n}\right)-\sigma\left(T_{n}\right)\right\|_{2}<\left\|\psi\left(T_{n}\right)-\sigma\left(T_{n}\right)\right\| \cdot m(I)=\varepsilon$. Hence, for any $n \geqq N_{1}$, $\left\|\sigma\left(T_{n}\right)-\sigma(T)\right\|_{2} \leqq\|\sigma(T)-\psi(T)\|_{2}+\left\|\psi(T)-\psi\left(T_{n}\right)\right\|_{2}+\left\|\psi\left(T_{n}\right)-\sigma\left(T_{n}\right)\right\|_{2}<\varepsilon+\varepsilon+\varepsilon=3 \varepsilon$.

Hence the theorem is proved.

We shall now deduce Theorem 4.2 from Theorem 4.4. Let $x$ be any continuity point of the distribution function of $T$. Then by Theorem $4.4,\left\{P_{n}^{x}\right\}$ converges in measure and because of uniform boundedness, converges in the $L_{2}$-mean, to $P^{x}$, i.e., $m\left(\left(P_{n}^{x}-P^{x}\right)^{*}\left(P_{n}^{x}-P^{x}\right)\right) \rightarrow 0$. Since $\sigma$ is absolutely continuous with respect to $m$, it follows that $\sigma\left(\left(P_{n}^{x}-P^{x}\right)^{*}\left(P_{n}^{x}-P^{x}\right)\right) \rightarrow 0$. And this implies $\sigma\left(P_{n}^{x}-P^{x}\right) \rightarrow 0$, or $\sigma\left(P_{n}^{x}\right) \rightarrow \sigma\left(P^{x}\right)$, which completes the proof of theorem.

We conclude this section with a necessary and sufficient condition for weak convergence and convergence in measure to coincide. 
THEOREM 4.5. Let $\left\{T_{n}\right\}$ be an arbitrary sequence of selfadjoint operators converging weakly to a selfadjoint operator $T$. Then $\left\{T_{n}\right\}$ converges in measure to $T$ if and only if $T=c I$ for some real constant $c$.

Proof. First we shall show that the given condition is sufficient. Let $T=c I$ for some real $c$. We shall prove that $\left\{T_{n}\right\}$ converges in measure to $T$. Let $F_{n}$ be the distribution function of $T_{n}$ and $F$ that of $T$. It is easy to see that $F(x)=0$, if $x<c$, and $F(x)=1$ if $x \geqq c$. Also any point $(y \neq c)$ is a continuity point of $F$. Corresponding to $(-\infty, y]$, let $P_{n}^{y}$ denote the spectral projection of $T_{n}$ and $P^{y}$ that of $T(n=1,2, \ldots)$. When $y<c, P^{y}=0$, and when $y \geqq c, P^{y}=I$. In view of weak convergence, $m\left(P_{n}^{y}\right) \rightarrow$ $m\left(P^{y}\right)=0=P^{y}$ whenever $y<c$, i.e., $\left\{P_{n}^{y}\right\}$ converges in measure to $0=P^{y}$ whenever $y<c$. Whenever $y>c, m\left(P^{y}\right)=1$, so that $m\left(P_{n}^{y}\right) \rightarrow m\left(P^{y}\right)=1$, which implies that $\left\{P_{n}^{y}\right\}$ converges in measure to $I$. Thus, for any arbitrary continuity point $y$ of $F$, $\left\{P_{n}^{y}\right\}$ converges in measure to $P^{y}$. Hence by Theorem $4.4,\left\{T_{n}\right\}$ converges in measure to $T$.

CONVERSE. We shall give an example of a sequence of projections converging weakly to a projection, but still not converging in measure.

Let $\alpha$ be a continuous finite factor and $m$ the faithful normal trace on $\alpha$ with $m(I)=1$. Let $P, Q, R$, and $S$ be four mutually orthogonal projections in $\alpha$, with $m(P)=\frac{1}{4}=m(Q)=m(R)=m(S)$. Let $\left\{A_{n}\right\}$ be a decreasing sequence of projections contained in $Q$, such that $m\left(A_{n}\right) \rightarrow 0$. Let $R_{n}=P+A_{n}$. Then $m\left(R_{n}\right) \rightarrow \frac{1}{4}=m(S)$ and $m\left(R_{n}^{\perp}\right) \rightarrow \frac{3}{4}=m\left(S^{\perp}\right)$. Let $F$ be the distribution function of $S$ and $F_{n}$ that of $R_{n}$. All the involved operators being projections, the spectrum of each one of them is concentrated at the two points 0 and 1 . Thus, for any $x$ with $x<0, F_{n}(x)=0=F(x)$. For $0 \leqq x<1, F_{n}(x)=m\left(R_{n}^{\perp}\right) \rightarrow \frac{3}{4}=m\left(S^{\perp}\right)$ and $F(x)=m\left(S^{\perp}\right)$. For $x \geqq 1, F_{n}(x)=1$ $=F(x)$. And any point $x$ other than 0 and 1 is a continuity point of $F$. Hence for any such $x$ (in fact for all $x$ ), $F_{n}(x) \rightarrow F(x)$. But $\left\{R_{n}\right\}$ cannot converge in measure to $S$. For, if it does, then $\left\{R_{n} \cdot S\right\}$ will converge in measure to $S \cdot S=S$. But $R_{n} \cdot S=0$ for all $n$, while $S \neq 0$. Since $S \neq c$. I for any $c$, this counterexample completes the proof.

CoRollary 4.3. Let $\left\{T_{n}\right\}$ converge in measure to cI. Let $\Phi$ be a real, continuous function (not necessarily expressible as the sum of a finite number of monotonic continuous functions). Then $\left\{\Phi\left(T_{n}\right)\right\}$ converges in measure to $d I$, where $d=\Phi(c)$.

REMARK. This corollary is nontrivial and does not immediately follow from the definition of convergence in measure.

Proof. First we shall establish the following proposition:

"Let $\left\{T_{n}\right\}$ converge weakly to $T$. Then $\left\{\Phi\left(T_{n}\right)\right\}$ converges weakly to $\Phi(T)$."

To prove this, let $g$ denote any real, bounded and continuous function. Let $h$ denote the composite map $g . \Phi$. Clearly $h$ is bounded and continuous. Now $m\left(g(\Phi)\left(T_{n}\right)\right)=m\left(h\left(T_{n}\right)\right) \rightarrow m(h(T))$ since $\left\{T_{n}\right\}$ converges to $T$ weakly. And the arbitrariness of $g$ implies that $\left\{\Phi\left(T_{n}\right)\right\}$ converges weakly to $\Phi(T)$. Hence the proposition.

Now, the corollary is an immediate consequence of Theorem 4.5 and the above proposition. 
5. Some dominated convergence theorems. In this section we shall state and prove some dominated convergence theorems which, in the case of a finite gage space, are stronger than the corresponding results of Stinespring [10].

Definitions. A sequence $\left\{A_{n}\right\}$ of operators is said to be $U$-continuous if, given any $\varepsilon>0$, there exists a $\delta>0$, such that for any projection $P$ with $m(P)<\delta$, one has $\left|m\left(A_{n} P\right)\right|<\varepsilon$ for all $n$, and $V$-continuous if $\left\|A_{n} P\right\|_{2}<\varepsilon$ for all $n$. Our definition of gross convergence is the same as that of Stinespring [10, pp. 23 and 26]. It is known [10, p. 32] that convergence in measure always implies gross convergence and that these two concepts are equivalent when the gage of the identity is finite. The orthogonal complement of any projection $P$ will be denoted either by $P^{\perp}$ or by $I-P$. For any operator $T, \operatorname{Re} T$ will denote $\left(T+T^{*}\right) / 2$ and $\operatorname{Im} T$ will denote $\left(T-T^{*}\right) / 2 i$.

For a sequence $\left\{A_{n}\right\}$ of operators converging grossly to an operator $A$, the following two theorems have been proved by Stinespring (without assuming the finiteness of the gage).

THEOREM 5.1 [10, P. 29]. If there exists a nonnegative integrable operator $T$ such that $-T \leqq \operatorname{Re} A_{n} \leqq T$, and $-T \leqq I_{m} A_{n} \leqq T$ for all $n$, then $A_{n} \rightarrow A$ in the $L_{1}$-mean.

THEOREM 5.2 [10, P. 30, AND THE REMARK IN P. 31]. If there exists a nonnegative integrable $T$ with $\left(\operatorname{Re} A_{n}\right)^{2} \leqq T$ and $\left(\operatorname{Im} A_{n}\right)^{2} \leqq T$ for all $n$, then $A_{n} \rightarrow A$ in the $L_{2}$-mean.

However, even in finite gage spaces there are cases which are not covered by these theorems, i.e., one can construct a sequence $\left\{A_{n}\right\}$ of nonnegative integrable operators converging in measure to an integrable operator $A$, and $m\left(A_{n}\right) \rightarrow m(A)$; but there does not exist any integrable operator $T$ with $A_{n} \leqq T$ for all $n$.

E.g., let $\alpha$ be a continuous finite factor, and let $m$ be the faithful, normal trace on $\alpha$ with $m(I)=1$. Let $P_{1}$ be a projection with $m\left(P_{1}\right)=1 / 2$. Let $P_{2}$ be a projection contained in $I-P_{1}$ with $m\left(P_{2}\right)=1 / 2^{2}, \ldots$, and in general let $P_{n}$ be a projection contained in $I-P_{1}-\cdots-P_{n-1}$ with $m\left(P_{n}\right)=1 / 2^{n}$. Let $A_{n}=2^{n} / n$. $P_{n}$. Each $A_{n}$ is integrable and $\left\{A_{n}\right\}$ converges in measure and hence grossly to zero and $m\left(A_{n}\right)=1 / n$ $\rightarrow 0=m(0)$. If possible let there exist a nonnegative operator $T$ such that $A_{n} \leqq T$ for all $n$. Then it is easy to see that $m(T) \geqq m\left(T P_{1}\right)+\cdots+m\left(T P_{n}\right) \geqq \sum_{k=1}^{n} 1 / k$ for all $n$, which shows that $T$ cannot be integrable. In order to cover such exceptional cases also, we shall state and prove the following theorems and show how they apply to the above example.

In what follows, $\left\{T_{n}\right\}$ will denote an arbitrary sequence of operators converging in measure to an operator $T$, and $\left\{S_{n}\right\}$ will denote an arbitrary sequence of nonnegative integrable operators converging in measure to an integrable operator $S$, and such that $m\left(S_{n}\right) \rightarrow m(S)$.

THEOREM 5.3. If, corresponding to each $n,-S_{n} \leqq \operatorname{Re} T_{n} \leqq S_{n}$ and $-S_{n} \leqq \operatorname{Im} T_{n} \leqq S_{n}$, then $T_{n} \rightarrow T$ in the $L_{1}$-mean. 
THEOREM 5.4. If, corresponding to each $n,\left(\operatorname{Re} T_{n}\right)^{2} \leqq S_{n}$, and $\left(\operatorname{Im} T_{n}\right)^{2} \leqq S_{n}$, then $T_{n} \rightarrow T$ in the $L_{2}$-mean.

REMARK. In the case of a finite gage space, these results are stronger than the corresponding results of Stinespring (Theorems 5.1 and 5.2 mentioned at the beginning of this section). Some dominated convergence theorems in arbitrary gage spaces have also been proved in [5].

LEMMA 5.1. Let $\left\{A_{n}\right\}$ be a sequence of nonnegative integrable operators converging in measure to an operator $A$, and let $m\left(A_{n}\right) \rightarrow m(A)$. Then $A_{n} \rightarrow A$ in the $L_{1}$-mean.

Proof. This lemma is well known in the commutative case. However, the method by which it is proved in standard textbooks on probability theory, [3, p. 140, Problem 17] does not directly extend to this general case, for which the proof is as follows. Let an arbitrary positive number $\varepsilon$ be given. For any $n$, let $C_{n}=A-A_{n}$, and $G_{n}^{\varepsilon}, F_{n}^{\varepsilon}$, and $H_{n}^{\varepsilon}$ denote the spectral projections of $C_{n}$ corresponding to the intervals $[\varepsilon, \infty),(-\infty,-\varepsilon]$, and $(-\varepsilon,+\varepsilon)$ respectively. It is easily seen that $H_{n}^{\varepsilon}$ is the spectral projection of $\left|C_{n}\right|$ corresponding to the interval $[0, \varepsilon)$. Now

$$
C_{n}=C_{n} G_{n}^{\varepsilon}+C_{n} F_{n}^{\varepsilon}+C_{n} H_{n}^{\varepsilon} \cdots
$$

and

$$
\left|C_{n}\right|=C_{n} G_{n}^{\varepsilon}-C_{n} F_{n}^{\varepsilon}+\left|C_{n}\right| H_{n}^{\varepsilon} \cdots
$$

Hence $0 \leqq m\left(C_{n} G_{n}^{\varepsilon}\right) \leqq m\left(G_{n}^{\varepsilon} A G_{n}^{\varepsilon}\right)$. Since $\left\{C_{n}\right\}$ converges in measure to zero, $m\left(G_{n}^{\varepsilon}\right) \rightarrow 0$ as $n \rightarrow \infty$, and since $A$ is integrable, this implies that $m\left(A G_{n}^{\varepsilon}\right) \rightarrow 0$. Hence $m\left(C_{n} G_{n}^{\varepsilon}\right)$ $\rightarrow 0$. Again $m\left(\left|C_{n}\right| H_{n}^{\varepsilon}\right) \leqq\left\|\left|C_{n}\right| \cdot H_{n}^{\varepsilon}\right\|=\varepsilon$, and similarly $\left|m\left(C_{n} H_{n}^{\varepsilon}\right)\right|<\varepsilon$. As $m\left(C_{n}\right) \rightarrow 0$, it follows from (1) that

$$
\limsup _{n \rightarrow \infty}\left|m\left(C_{n} F_{n}^{\varepsilon}\right)\right| \leqq \varepsilon
$$

Hence,

$$
\limsup _{n \rightarrow \infty} m\left(\left|C_{n}\right|\right) \leqq \varepsilon+\varepsilon=2 \varepsilon .
$$

$\varepsilon$ being arbitrary, the desired result follows.

LEMMA 5.2. Let $\left\{A_{n}\right\}$ be any sequence of nonnegative, integrable operators. Then $A_{n} \rightarrow A$ in the $L_{1}$-mean if and only if $\left\{A_{n}\right\}$ is $U$-continuous and converges in measure to $A$.

Proof. If $A_{n} \rightarrow A$ in the $L_{1}$-mean, then it is known that it converges to $A$ in measure, and it is easily verified that $\left\{A_{n}\right\}$ is $U$-continuous. For the converse, let an arbitrary positive number $\varepsilon$ be given. By assumption there exists a " $\delta$ " $>0$ such that, for any projection $P$ with $m(P)<\delta$, one has $m\left(A_{n} P\right)<\varepsilon$ for all $n$. The sequence $\left\{P A_{n} P\right\}$ converges in measure to $P A P$. The involved operators being nonnegative, it follows by the noncommutative version of Fatou's Lemma $[9, \mathrm{p}$. 31] that $m(P A P) \leqq \lim \inf _{n \rightarrow \infty} m\left(P A_{n} P\right) \leqq \varepsilon$, i.e., $m(A P) \leqq \varepsilon$. Let $P_{n}$ be the spectral projection of $\left|A_{n}-A\right|$ corresponding to the interval $[0, \varepsilon)$ and $Q_{n}=I-P_{n}$. Then $m\left(P_{n}\right) \rightarrow 1$. 
So, for all $n \geqq$ some positive integer $N, m\left(P_{n}\right) \geqq 1-\delta$. Thus for any $n \geqq N$,

$$
\begin{aligned}
\left\|A_{n}-A\right\|_{1} & =m\left(\left|A_{n}-A\right|\right) \\
& =m\left(\left|A_{n}-A\right| P_{n}\right)+m\left(\left|A_{n}-A\right| Q_{n}\right) \\
& \leqq \varepsilon+m\left(\left|A_{n}-A\right| Q_{n}\right) .
\end{aligned}
$$

Let $U_{n}$ and $W_{n}$ denote the spectral projection of $A_{n}-A$ corresponding to $[\varepsilon, \infty)$ and $(-\infty,-\varepsilon]$ respectively. Then $Q_{n}=U_{n}+W_{n}$, so that $m\left(U_{n}\right) \leqq \delta$ and $m\left(W_{n}\right) \leqq \delta$. And

$$
\begin{aligned}
m\left(\left|A_{n}-A\right| Q_{n}\right) & =m\left(\left(A_{n}-A\right) U_{n}\right)-m\left(\left(A_{n}-A\right) V_{n}\right) \\
& \leqq\left|m\left(A_{n} U_{n}\right)\right|+\left|m\left(A U_{n}\right)\right|+\left|m\left(A_{n} V_{n}\right)\right|+\left|m\left(A V_{n}\right)\right| \\
& \leqq \varepsilon+\varepsilon+\varepsilon+\varepsilon=4 \varepsilon .
\end{aligned}
$$

Hence $\left\|A_{n}-A\right\|_{1} \leqq \varepsilon+4 \varepsilon=5 \varepsilon$. $\varepsilon$ being arbitrary, the desired result follows.

LEMMA 5.3. For any square-integrable operator $A$, and $\varepsilon>0$, one can find $a$ " $\delta ">0$, such that for any projection $P$ with $m(P)<\delta$, one has $\|A P\|_{2}<\varepsilon$.

Proof. " $A$ " being square-integrable, there exists a bounded operator $B$ with $\|A-B\|_{2}<\varepsilon / 2$. Let $\|B\|=k$, let $\delta=\varepsilon / 2 k$, and $P$ any projection with $m(P)<\delta$. Then $\|B P\|_{2} \leqq\|B\| \cdot m(P) \leqq k \cdot \varepsilon / 2 k=\varepsilon / 2$. As $\|A P-B P\|_{2}<\varepsilon / 2$, it follows that $\|A P\|_{2}$ $\leqq \varepsilon / 2+\varepsilon / 2=\varepsilon$. Hence the lemma.

Lemma 5.4. For any square-integrable operator $A$ and projection $P$,

$$
m\left(|A|^{2} P\right)=\left(\|A P\|_{2}\right)^{2} .
$$

\section{Proof.}

$$
\begin{aligned}
\left(\|A P\|_{2}\right)^{2} & =m(A P)^{*}(A P) \\
& =m\left(\left(P A^{*}\right)(A P)\right) \\
& =m\left(P\left(A^{*} A\right) P\right) \\
& =m\left(P|A|^{2} P\right) \\
& =m\left(|A|^{2} P\right) .
\end{aligned}
$$

LEMMA 5.5. Let $\left\{A_{n}\right\}$ be a sequence of square-integrable operators. Then $A_{n} \rightarrow A$ in the $L_{2}$-mean, if and only if $\left\{A_{n}\right\}$ converges in measure to $A$, and is also $V$-continuous.

Proof. Let $A_{n} \rightarrow A$ in the $L_{2}$-mean. Then clearly $\left\{A_{n}\right\}$ converges in measure to $A$. Also, since $A_{n}-A$ and $A_{n}$ are square-integrable, it follows that $A$ is squareintegrable. By Lemma 5.3 , there exists a " $\delta$ " such that $\|A P\|_{2}<\varepsilon / 2$, for all projections $P$ with $m(P)<\delta_{1}$. As $A_{n} \rightarrow A$ in the $L_{2}$-mean, there exists a positive integer $N$, with $\left\|A_{n}-A\right\|_{2}<\varepsilon / 2$ for all $n \geqq N$. Hence $\left\|A_{n} P\right\|_{2}<\varepsilon$ for all $n>N$. Also $A_{1}, \ldots, A_{N}$ being each square-integrable, and $N$ being finite, there exists a $\delta_{2}$ such that for any projection $P$ with $m(P)<\delta_{2}$, we have $\left\|A_{i} P\right\|_{2}<\varepsilon / 2, i=1,2, \ldots, N$. Let $\delta=\min \left(\delta_{1}, \delta_{2}\right)$. Then for any projection $P$ with $m(P)<\delta$, we have $\left\|A_{i} P\right\|_{2}<\varepsilon$, $i=1,2, \ldots$, which shows that $\left\{A_{n}\right\}$ is $V$-continuous. For the converse note that, since $\left\{A_{n}\right\}$ converges in measure to $A,\left\{\left|A_{n}\right|^{2}\right\}$ converges in measure to $|A|^{2}$. Let an 
arbitrary positive number $\varepsilon$ be given. Since $\left\{A_{n}\right\}$ is $V$-continuous, it follows that there exists a $\delta>0$, such that for any projection $P$ with $m(P)<\delta,\left\|A_{n} P\right\|_{2}<\varepsilon$ for all $n$. Hence $\left(\left\|A_{n} P\right\|_{2}\right)^{2}=m\left(\left|A_{n}\right|^{2} P\right)<\varepsilon^{2}$. By Fatou's Lemma, it follows that $m\left(|A|^{2} P\right)$ $<\varepsilon^{2}$, i.e., $\left(\|A P\|_{2}\right)^{2}<\varepsilon^{2}$. As $\left\{A_{n}\right\}$ converges in measure to $A$, there exists a sequence $\left\{Q_{n}\right\}$ of projections such that $\left\|\left(A_{n}-A\right) Q_{n}\right\|<\varepsilon$ for all $n$, and $m\left(Q_{n}\right) \rightarrow 1$. Hence for all $n \geqq$ some positive integer $N, m\left(Q_{n}^{\perp}\right)<\delta$, so that for any $n \geqq N$,

$$
\begin{aligned}
\left\|A_{n}-A\right\|_{2} & \leqq\left\|\left(A_{n}-A\right) Q_{n}\right\|_{2}+\left\|\left(A_{n}-A\right) Q_{n}^{\perp}\right\|_{2} \\
& \leqq \varepsilon+\left\|A_{n} Q^{\perp}\right\|_{2}+\left\|A Q_{n}^{\perp}\right\|_{2} \\
& \leqq \varepsilon+\varepsilon+\varepsilon=3 \varepsilon
\end{aligned}
$$

Hence the lemma is proved.

Proof of Theorem 5.3. It suffices to prove the theorem assuming each $T_{n}$ to be selfadjoint, as the general case is reducible to this. Since $-S_{n} \leqq T_{n} \leqq S_{n}$, it follows that $-S \leqq T \leqq S$, which shows that $T$ is integrable. Again, it follows by Lemma 5.1 that $S_{n} \rightarrow S$ in the $L_{1}$-mean. Therefore it also follows that $\left\{S_{n}\right\}$ and hence $\left\{T_{n}\right\}$ are $U$-continuous. Thus, given an arbitrary positive number $\varepsilon$, there exists a $\delta>0$, such that for any projection $P$ with $m(P)<\delta$, one has $\left|m\left(T_{n} P\right)\right|<\varepsilon$ for all $n$. Let $G_{n}$ and $F_{n}$ denote the spectral projections of $T_{n}-T$ corresponding to the intervals $[\varepsilon / 2, \infty)$ and $(-\infty,-\varepsilon / 2]$. Let $K_{n}=I-\left(G_{n}+F_{n}\right)$. Then $K_{n}$ is the spectral projection of $\left|T_{n}-T\right|$ corresponding to $[0, \varepsilon / 2)$. In view of convergence in measure, $m\left(K_{n}\right) \rightarrow 1$ as $n \rightarrow \infty$; hence there exists a positive integer $N$ such that for $n \geqq N, m\left(K_{n}\right)>1-\varepsilon$, so that $m\left(G_{n}\right)$ and $m\left(F_{n}\right)$ are both $<\delta$. Now, for any $n \geqq N$,

$$
\begin{aligned}
\left\|T_{n}-T\right\|_{1} & =m\left(\left|T_{n}-T\right|\right) \\
& =m\left(\left|T_{n}-T\right| K_{n}\right)+m\left(\left|T_{n}-T\right| G_{n}\right)+m\left(\left|T_{n}-T\right| F_{n}\right) \\
& \leqq \varepsilon+m\left(\left|T_{n}-T\right| G_{n}\right)+m\left(\left|T_{n}-T\right| F_{n}\right) \\
& =\varepsilon+m\left(\left(T_{n}-T\right) G_{n}\right)-m\left(\left(T_{n}-T\right) F_{n}\right) \\
& \leqq \varepsilon+\left|m\left(T_{n} G_{n}\right)\right|+\left|m\left(T G_{n}\right)\right|+\left|m\left(T_{n} F_{n}\right)\right|+\left|m\left(T F_{n}\right)\right| \\
& \leqq \varepsilon+\varepsilon+\varepsilon+\varepsilon+\varepsilon=5 \varepsilon .
\end{aligned}
$$

Hence $T_{n} \rightarrow T$ in the $L_{1}$-mean. The general case follows by applying the previous result separately to the sequences $\left\{\operatorname{Re} T_{n}\right\}$ and $\left\{\operatorname{Im} T_{n}\right\}$.

Proof of Theorem 5.4. To begin with, let us assume that each $T_{n}$ is selfadjoint. As $S_{n} \rightarrow S$ in the $L_{1}$-mean, it follows that $\left\{S_{n}\right\}$ is $U$-continuous. Since $T_{n}^{2} \leqq S_{n}$, $\left\{T_{n}^{2}\right\}$ is also $U$-continuous. Further, for any projection $P, m\left(T_{n}^{2} P\right)=\left(\left\|T_{n} P\right\|_{2}\right)^{2}$ so that the sequence $\left\{T_{n}\right\}$ is $V$-continuous. Also $\left\{T_{n}\right\}$ converges in measure to $T$. Hence by Lemma $5.5, T_{n} \rightarrow T$ in the $L_{2}$-mean.

The general case can be proved by applying the previous result, separately to $\left(\operatorname{Re} T_{n}\right)^{2}$ and $\left(\operatorname{Im} T_{n}\right)^{2}$.

We shall now show how our theorems apply to the example given at the beginning of this section. Let $A_{n}, P_{n}, \ldots$, etc., be as in that example. Define $B_{n}=A_{n}+P_{n}^{\perp}$. Then $\left\{B_{n}\right\}$ converges in measure to $I$ and $m\left(B_{n}\right) \rightarrow 1$ and it follows by Theorem 5.3 that $A_{n} \rightarrow 0$ in the $L_{1}$-mean. 
6. Applications to operator-entropy. I. Let $\left\{T_{n}\right\}$ be a sequence of nonnegative, square-integrable operators, converging in the $L_{2}$-mean to an operator $T$. Then the operator-entropy of $T_{n}\left(=-T_{n} \log T_{n}\right)$, tends in the $L_{1}$-mean, to the operatorentropy of $T(=-T \log T)$, and in particular, the numerical entropy of $T_{n}$ $\left(=m\left(-T_{n} \log T_{n}\right)\right)$ tends to the numerical entropy of $T$.

Proof. Let $\sigma(\lambda)=\lambda \log \lambda$, where $\sigma(0)$ is defined to be zero. Hence $\sigma(\lambda)$ is continuous in the closed unit interval so that there exists a positive constant $c$, such that $-c<\sigma(\lambda)<c$, whenever $0 \leqq \lambda \leqq 1$. Hence it follows that for any nonnegative operator $A$

$$
-A^{2}-c I \leqq A \log A \leqq A^{2}+c I .
$$

Since $\left\{T_{n}\right\}$ converges in the $L_{2}$-mean to $T$, it converges in measure to $T$. Hence $\left\{T_{n}^{2}\right\}$ converges in measure to $T^{2}$. Also as $\left\|T_{n}-T\right\|_{2} \rightarrow 0$, it follows that $m\left(T_{n}^{2}\right)$ $\rightarrow m\left(T^{2}\right)$. Thus if we set $S_{n}=T_{n}^{2}+c I$, and $S=T^{2}+c I$, then $\left\{S_{n}\right\}$ is a sequence of nonnegative integrable operators converging in measure to a nonnegative integrable operator $S$ and also $m\left(S_{n}\right) \rightarrow m(S)$. Moreover, $-S_{n} \leqq T_{n} \log T_{n} \leqq S_{n}$ for each $n$. As $\left\{T_{n}\right\}$ converges in measure to $T$, it follows by Theorem 2 of $\S 2$ that $\left\{T_{n} \log T_{n}\right\}$ converges in measure to $T \log T$. Now as a consequence of our dominated convergence theorem, it follows that $\left\{T_{n} \log T_{n}\right\}$ converges in the $L_{1}$-mean to $T \log T$.

Before proceeding further, we shall prove a lemma.

Lemma 6.1. Let $\beta$ be any ring contained in $\alpha$. Let $A$ be any square-integrable operator and let $K=E[A \mid \beta]$ denote the conditional expectation of $A$, given $\beta$ in the sense of Umegaki [11]. Then one has $\|K\|_{p} \leqq\|A\|_{p}, p=1,2$.

Proof. We shall first show this for $p=1$. Note that for any $U$ in $\beta, m(A U)$ $=m(K U)$. Let $U_{1}$ denote the unit sphere of $\alpha$ and $U_{2}$ that of $\beta$. Then,

$$
\begin{aligned}
\|K\|_{1} & =\sup _{U \operatorname{in} U_{2}}(|m(K U)|) \\
& =\sup _{U \operatorname{in} U_{2}}(|m(A U)|) \\
& \leqq \sup _{U \text { in } U_{1}}(|m(A U)|) \\
& =\|A\|_{1} .
\end{aligned}
$$

For $p=2: G=L_{2}(H, \alpha, m)$ is a Hilbert space, of which $N=L_{2}(H, \beta, m)$ is a closed subspace, " $A$ " is an element of $G$, and $K$ is its projection on the subspace $N$. Hence the norm of $K$ regarded as an element of $N\left(=\|K\|_{2}\right)$ is less than or equal to the norm of $A$, regarded as an element of $G\left(=\|A\|_{2}\right)$. Thus $\|K\|_{2} \leqq\|2\|_{2}$.

Using these two results, we can rewrite as follows the two dominated convergence theorems which we have proved:

THEOREM 6.1. Let all the assumptions of Theorem 5.3 (Theorem 5.4) be satisfied. Then, in the notation of Theorem 5.3 (Theorem 5.4), $E\left(T_{n} \mid \beta\right) \rightarrow E(T \mid \beta)$ in the $L_{1}$-mean (in the $L_{2}$-mean). 
II. Let $T$ be any nonnegative square-integrable operator and $R$ any bounded selfadjoint operator. Let $\beta$ be the ring generated by $R$, and let $S=E(T \mid \beta)$. Then the number $m(T \log T)-m(S \log S)$ is defined by Nakamura and Umegaki [4] to be the information about $T$ contained in $R$. This we shall denote by $I(T ; R)$. $I(T ; R)$ is finite, $T$ being square-integrable.

As another application of our theorems, we state the following result:

Let $\left\{T_{n}\right\}$ be a sequence of nonnegative, and square-integrable operators, converging in the $L_{2}$-mean to $T$. Then, in the above notation, $I\left(T_{n} ; R\right) \rightarrow I(T ; R)$.

Proof. Let $E\left(T_{n} \mid \beta\right)=S_{n}$ and $E(T \mid \beta)=S$. Then, by Lemma 6.1, $S_{n} \rightarrow S$ in the $L_{2}$-mean. Hence, by an earlier result, $m\left(T_{n} \log T_{n}\right) \rightarrow m(T \log T)$ and $m\left(S_{n} \log S_{n}\right)$ $\rightarrow m(S \log S)$. Thus

$$
\begin{aligned}
I\left(T_{n} ; R\right) & =m\left(T_{n} \log T_{n}\right)-m\left(S_{n} \log S_{n}\right) \\
& \rightarrow m(T \log T)-m(S \log S) \\
& =I(T ; R) .
\end{aligned}
$$

ACKNOwLedgements. The author is grateful to Professor I. E. Segal for having gone through the material in this paper and given his comments; to Professor Umegaki for having gone through parts of this paper and suggested Example 3 in $\S 2$; and to Dr. Sethuraman and Mr. U. S. R. Murthy for valuable suggestions for improving the presentation of this paper. He also profited from conversations with Mr. Viswanath and suggestions of the referee.

\section{REFERENCES}

1. B. V. Gnedenko and A. N. Kolmogorov, Limit distributions for sums of independent random variables, Addison-Wesley, Reading, Mass., 1954.

2. A. N. Kolmogorov, Foundations of the theory of probability, Chelsea, New York, 1956.

3. M. Loève, Probability theory, 3rd ed., Van Nostrand, New York, 1963.

4. M. Nakamura and H. Umegaki, $A$ note on the entropy for operator algebra, Proc. Japan Acad. 37 (1961), 149-151.

5. A. R. Padmanabhan, Some dominated convergence theorems in a von Neumann algebra, Proc. Japan Acad. 42 (1966), 347-350.

6. I. E. Segal, A non-commutative extension of abstract integration, Ann. of Math. (2) 57 (1953), 401-457.

7. - Tensor algebras over Hilbert spaces. II, Ann. of Math. (2) 63 (1956), 160-175.

8. - Distributions in Hilbert space and canonical systems of operators, Trans. Amer. Math. Soc. 88 (1958), 12-41.

9. D. Shale and W. F. Stinespring, States of the Clifford algebra, Ann. of Math. (2) 80 (1964), 365-381.

10. W. F. Stinespring, Integration theorems for gages and duality theorems for unimodular groups, Trans. Amer. Math. Soc. 90 (1959), 15-56.

11. H. Umegaki, Conditional expectation in an operator algebra, Tôhoku Math. J. 6 (1954), 177-181.

INDIAN STATISTICAL INSTITUTE

Calcutta-35, India

INDIANA UNIVERSITY,

BLOOMINGTON, INDIANA 